\title{
Modern Portfolio Theory, Digital Portfolio Theory and Intertemporal Portfolio Choice
}

\author{
C. Kenneth Jones \\ Portfolio Networks, Gainesville, USA \\ Email: kenjones@portfolionetworks.com
}

How to cite this paper: Jones, C.K. (2017) Modern Portfolio Theory, Digital Portfolio Theory and Intertemporal Portfolio Choice. American Journal of Industrial and Business Management, 7, 833-854.

https://doi.org/10.4236/ajibm.2017.77059

Received: April 20, 2017

Accepted: July 3, 2017

Published: July 6, 2017

Copyright $\odot 2017$ by author and Scientific Research Publishing Inc. This work is licensed under the Creative Commons Attribution International License (CC BY 4.0).

http://creativecommons.org/licenses/by/4.0/

\begin{abstract}
The paper compares three portfolio optimization models. Modern portfolio theory (MPT) is a short-horizon volatility model. The relevant time horizon is the sampling interval. MPT is myopic and implies that investors are not concerned with long-term variance or mean-reversion. Intertemporal portfolio choice is a multiple period model that revises portfolios continuously in response to relevant signals to reduce variance of terminal wealth over the holding period. Digital portfolio theory (DPT) is a non-myopic, discrete time, long-horizon variance model that does not include volatility. DPT controls mean-reversion variances in single period solutions based on holding period and hedging and speculative demand.
\end{abstract}

\section{Keywords}

Portfolio Theory, Portfolio Optimization, Mean-Reversion Risk, Long-Horizon Risk, Portfolio Diversification

\section{Introduction}

Advances in signal processing in recent years have resulted in a "golden age of digital signal processing" characterized by stability and reliability. In addition to revolutionizing communication systems and electronic devices, this technology is now taking on a new role of understanding human-centric signals and information. In this same period, the field of finance has enjoyed an age of volatility characterized by high leverage and uncertainty. This paper looks at the potential benefit of the digital revolution when applied to portfolio theory and compares it with existing models. Markowitz [1] provides the basic framework for shortterm mean variance portfolio selection but little practical guidance for long-term investors. Modern Portfolio Theory (MPT) is single period myopic and offers no solution to the empirical long-horizon portfolio problem. The Merton [2] mul- 
ti-period non-myopic model provides the dynamic solution to the two-asset long-horizon, portfolio choice problem. Intertemporal portfolio choice gives basic insight into finding long-term optimal non-myopic solutions based on hedging and speculative demands when returns are not random. Practical implementation of intertemporal portfolio theory, however, is prohibitively difficult. More recently, Digital Portfolio Theory (DPT) Jones [3] is significantly easier to solve than MPT and provides a non-myopic, single period, long-horizon solution. DPT incorporates mean-reversion risk and allows hedging and speculative demands based on holding period. Mean-reversion risk is quantified using low-frequency digital signal processing (DSP). I examine the characteristics of DPT in relation to MPT and to intertemporal portfolio choice. I address questions of how hedging and speculative demand for mean-reversion risks, and holding periods influence solutions.

\section{MPT, DPT and Intertemporal Portfolio Choice}

Classical Markowitz portfolio selection finds portfolio weights based on first moments and a parametric representation of second moments assuming no predictable time variation. Mean variance MPT assumes investment opportunities are constant, or returns are independently distributed over time (IID). The static mean variance solution is myopic and does not consider events beyond the present period. The nonlinear mean variance optimization is ill-conditioned because solution methodologies introduce problems such as; estimation error amplification, extreme positions, unstable solutions, excessive sensitivity to mean and covariance estimates, a tendency to find solutions that diverge from pricing model recommendations, and no solutions for large universes do to a singular covariance matrix.

Portfolio theory evolved from the myopic mean variance paradigm to the multi-period or continuous-time models of Samuelson [4] and Merton [5] [6]. Long-horizon problems focus on finding portfolio weights with shifting investment opportunities over multiple periods. Merton's [2] dynamic portfolio choice solutions are different from single period solutions due to mean reverting return distributions and due to periodic revision or rebalancing. The portfolio of the multi-period investor differs from the single period investor because of hedging and speculative demands. The Merton intertemporal maximizer takes into account the relation between current period returns and predicted future returns. Multiple period investors will hold more low correlated assets in the current period to hedge the possibility of lower expected returns in the future periods. Intertemporal portfolio choice finds dynamic multiple period solutions that adjust allocations to reduce the variance of terminal wealth. Hedging and speculative demands arise because of mean-reversion. When returns are predictable using a set of lagged state variables, investors who want optimal multi-period solutions have hedging demands that influence the composition of their equity portfolios. The Merton multi-period approach provides a useful economic intuition about optimal portfolio choice and risk. It has little value for investors seeking optimal 
portfolios from large asset universes. Portfolio managers do not use the Merton model due to its complexity, dependence on utility function parameters, and dependence on state variables and forecasting models. The solution to dynamic investment problems, to capture multi-period predictability in first and second moments, often require restrictive conditional models to find intertemporal dynamic programming solutions.

DPT is a long horizon paradigm based on a nonparametric representation of variances, covariances, and autocovariances and is solved with a linear programming (LP) solution. The DPT mean-variance-autocovariance problem allows multiple horizon hedging, or speculative demands based on holding period and has a static single period solution. Horizon effects result when returns have mean-reversions. Mean-reversions over different horizons influence the single period decision. DPT uses contributions to single period risk of multiple meanreversion lengths but uses no information about of the pattern of mean-reversions. Instead, it uses long-term forecasts of the level of mean-reversion risks. Unconditional hedging demand for mean-reversion risk occurs in single period DPT when the holding period is greater than or equal to the mean-reversion length. For example, an investor with a 2 -year holding period will have unconditional hedging demand for 6-month mean-reversion risk and 1-year mean-reversion risk but will have zero hedging demand for 4-year mean-reversion risk. Conditional speculative demand for mean-reversion risk occurs in DPT when an investor has a forecast of the cyclical period of a mean-reversion. For example, if we have a forecast that 4 -year mean-reversion is currently at a 4 -year peak we could speculate by reducing 4 -year mean-reversion risk. If we have a forecast that 6-month mean-reversion is at a bottom we can speculate by increasing 6month mean-reversion risk.

DPT defines the non-myopic asset allocation problem based on autocovariance and variance of mean reverting returns. In DPT hedging demand for risky assets is larger the longer the holding period because more mean-reversion lengths are included in the holding period. As the holding period gets shorter fewer of the mean-reversion lengths can be used to hedge. Hedging demand normally decreases and speculative demand increases as an investors holding period shortens. In the Jones [7] long horizon portfolio theory, unconditional horizon effects induced by unconditional autocorrelation in return or unconditional mean-reversion variance will result in different single period optimal portfolios compared to MPT. DPT has a static non-myopic solution that incorporates the probability of mean-reversions in the investment opportunity set. This allows hedging considerations in single period solutions to the long-horizon problem without relying on lagged forecasting models, or state variables. Since DPT finds solutions using linear programming, it does not have the distortions encountered in quadratic MPT solutions. Single period DPT solutions allow unconditional multi-period hedging demands for risky assets based on holding period and conditional speculative demand for mean-reversion risk based on forecasts of the stage of mean-reversions. 


\section{Literature Review}

To understand the mechanism used to optimize a portfolio over multiple periods in the presence of mean-reversion we need to examine prior research. The analysis of hedging demands originated with Merton and was followed by a large number of studies utilizing multiple period problems. These multiple period models often include both the portfolio selection and the consumption decision. The studies examine the multi-period portfolio choice problem in the presence of return predictability, frequently for a two-asset universe. Campbell and Viceira [8], Brandt [9], Ait-Sahalia and Brandt [10] propose dynamic intertemporal optimization solutions that capture mean-reversions in stock returns using AR models. Campbell and Viceira [8] use multi-period models with two assets, and discrete time AR (1) processes to describe mean reverting return predictability of the risky asset. They find that 20 to 50 percent of stock demand results from hedging demands. Allowing no short sales and using the dividend price ratio as the state variable, Barberis [11] finds that investors should normally be 100 percent invested in stocks. Brennan, Schwartz and Lagnado [12] and Barberis [11] analyze numerically the impact of myopic dynamic decision-making, while Kim and Omberg [13] and Liu [14] obtain exact analytical solutions for a range of continuous-time problems with predictability. Brandt [9], using the dividend yield as a forecasting state variable, formulates the single period problem when return distributions vary through time. Optimal portfolio choice is conditional on the state variable in the prior period. Brandt uses the investor's Euler equation to estimate portfolio allocation to stocks and finds small positive hedging demands that increase with horizon independent of rebalancing frequency. Intertemporal solutions provide intuition in limited settings but little practical asset allocation advice to managers who require an optimization criterion for selecting portfolios.

Many models focus on optimal dynamic multi-period policies based on conditional predictability of first moments. Ait-Sahalia and Brandt [10] define horizon effects as different portfolio choices for single period investors with different horizons based on unconditional or conditional autocorrelations or mean-reversions in returns. Campbell and Viceira [8] [15], Balduzzi and Lynch [16], Brandt [9], Chacko and Viceira [17], Barberis [11] and Lynch [18] find relatively large hedging demands. Ait-Sahalia and Brandt [10] unlike other studies find small hedging demands and even find negative hedging demand, with longterm investors allocating less wealth to risky securities than short-term investors. Lynch [18] uses Merton's multi-period continuous time model with dividend yield as the predictive state variable, to find lifetime asset allocation based on characteristics like size and book-to-market value. Lynch finds that investors should hold less high book-to-market early in life because of hedging demands. There is growing recognition that any viable method to finding long horizon optimal portfolios for a real asset universe will require finding a static problem that incorporates information about predictable return dynamics. Brandt and Santa Clara [19] attempt to restate the dynamic portfolio selection problem as a static 
problem by adding; artificial portfolios to the asset universe that reflect conditioning state variables, and timing portfolios that reflect the risky asset in each period. Then the Markowitz static single period mean variance quadratic solution identifies the optimal dynamic strategy. These studies point to the importance of applying hedging and speculative demands using multiple revisions with predictability within the holding period. In multiple period portfolio optimizations with mean reverting returns and with some prediction of the future opportunity set, hedging and speculative demand as proposed by intertemporal portfolio choice will result in higher return and lower risk than a myopic approach such as MPT.

\section{Modern Portfolio Theory}

MPT provides a useful diversification paradigm but ignores risks beyond one period ahead. The Markowitz solution gives the myopic demand or the investment policy of an investor who optimizes over the next period without regard for changes in the investment opportunity set. Myopic mean variance demand does not include intertemporal hedging. In a myopic strategy the optimal portfolio in the current revision period does not depend on the investment opportunities in future revision periods. The MPT solution is optimal when the investment opportunity set is constant, when returns are IID over time, or when investor's utility is logarithmic. With constant investor opportunities, investors will behave as single period optimizers. If the asset returns are IID, risky assets cannot hedge changes in investment returns, hedging demand is zero, and all risky asset demand is myopic. The dynamic problem reduces to the single period problem when returns are IID. The traditional approach of applying myopic mean variance optimization each period ignores mean-reversions and intertemporal hedging demands. Over a multiple period horizon, static buy-and-hold single period Markowitz solutions using multiple period returns are not optimal when portfolio revisions are possible each period, and return distributions have predictable time variation. Mean variance solutions exclude Merton's hedging components driven by predictive state variables. With predictable returns, intertemporal portfolio theory with multiple revisions will result in a lower variance of terminal wealth compared to the one period MPT solution.

\subsection{Problems with MPT}

The MPT database approach uses return time series to estimate means and covariance. It does not consider the composition of risk in terms of systematic (passive) risk, or unsystematic (active) risk, nor does it consider investment horizon risk. Capital market theory suggests that with a risk-free asset, two-fund separation applies and the tangent efficient portfolio will contain only systematic risk. Merton and Samuelson [20] point out "the mean variance model has interest because of its separation property." They develop an intertemporal portfolio theory in continuous time assuming IID return processes that has many of the properties of static mean variance theory. 
One difficulty with MPT is that correlations between securities are unreliable. To improve correlations and describe active and passive risk, model-based approaches find parameters by applying an asset pricing relation such as the CAPM, or APT and use benchmark returns to describe optimal portfolios. To give greater control over systematic sources of risk the covariance matrix is represented with multiple factors or scenarios that describe common sources of covariance and assumes that remaining variability is security specific and uncorrelated (Perold [21]). Another difficulty is that the covariance matrix is illconditioned, when $\mathrm{N}$ (the universe size) is the order of magnitude of the number of historical time series returns used in the estimation. Inverting the sample covariance matrix to find the solution amplifies estimation error. If the covariance matrix is not invertible ( $\mathrm{N}$ is larger than the time series length), an alternative risk model must be used.

The quadratic function that describes the weights in MPT magnifies estimation errors. Michaud [22] argues that classic mean variance optimizers are estimation error amplifiers. Matrix inversion, cross product, and squared decision variables, magnify estimation errors in weights. The covariance matrix is unstable. Terms grow exponentially with universe size increasing distortion. Efficient MPT weights are meaningful only for small universe solutions. In addition, historically estimated MPT may find inappropriate solutions or "unnatural portfolios" because expected returns are mean reverting rather than independent.

\subsection{Extreme and Unstable Positions, Estimation Error, and Pricing Models}

MPT solution weights tend to be extreme. Green and Hollifield [23] find that extreme weights result from the dominance of a single factor in the covariance matrix. MPT assumes that the parameters of the return generating process are know rather than estimated. It does not incorporate estimation error in the model parameters. For this reason, optimal portfolios are small with concentrated allocations. Research suggests that the relatively small portfolios found with long only MPT should be larger do to high volatility, large estimation error, weak correlations, and poor expected return forecasts. If we treat MPT as a statistical sampling problem, optimal portfolios must be larger. Frost and Savarino [24], and Jagannathan and $\mathrm{Ma}$ [25] use constraints to avoid extreme positions. To mitigate estimation errors, poor forecasts, and extreme weights, shrinkage methods are used to moderate sample means and covariances toward investor's expectations or towards values specified by a model such as the CAPM (Jobson and Korkie [26], Black and Litterman [27]). Estimates of means can be improved using shrinkage methods (e.g., Jobson and Korkie [28]). The covariance matrix can be improved using shrinkage e.g., Ledoit and Wolf [29]. Estimates can be improved with the combination of priors from alternative pricing models, or using data sources with information contained in returns (e.g., Treynor and Black [30], Black and Litterman [27] and Pastor and Stambaugh [31]).

Another problem is that MPT solution weights tend to be unstable. Instability 
is the result of sensitivity of the weights to small variations in the sample mean, variance, and correlation resulting from parameter uncertainty. The solution weights are subject to high levels of estimation error with correlations between securities estimated at low significance.

\section{Intertemporal Portfolio Theory}

\subsection{Assumptions}

Merton [5] [6] solves the consumption-portfolio choice problem in continuous time using expected utility maximization when security prices follow diffusion processes. Merton assumes changes in returns, investment opportunities, and state variables follow smooth and continuous diffusion processes. Merton's continuous time approach uses an infinitely small decision interval making multiple period problems necessary to find long-horizon solutions. Merton considers daily returns and trading to be short enough to satisfy the continuous-time assumption with a one-year holding period. The solution to the multi-period problem is a trading strategy that adjusts for shifting return distributions with new weights for each security in each period. Suppose we have a one-year holding period. We could estimate return annually and find the efficient frontier. However, if we continuously revise (daily) and have a forecast over the year, we will lower the variance of terminal wealth. In practice, monthly or quarterly revisions are used with holding periods of several years. Long revision periods, however, may violate the assumptions of continuous trading and continuous diffusion processes. In multiple period frameworks, non-myopic portfolio strategies depend on the investor's opportunity set in future periods. Multi-period Merton asset allocation problems are normally conditional, the investor uses a set of state variables at time $t$ to predict the return at time $t+1$. Merton shows that when the means and variances of returns are changing, dependent on conditional state variables or past returns, the intertemporal optimal portfolios differs from the static one-period Markowitz model. Since the investor's objective is to maximize terminal wealth while limiting the variance of terminal wealth, the multi-period investor will have a different optimal portfolio composition than an investor who holds the portfolio one period. With predictability, the portfolio composition in each daily, monthly or quarterly revision includes hedging and speculative demands that reflect predictable variation in the returns over the remaining holding period and beyond.

\subsection{Additive Myopic and Non-Myopic Risky Asset Demand}

In the Merton [5] [6] model, multi-period investors' value risky assets for both short-term return, and for intertemporal hedging against adverse shifts in investment opportunities. Shifts that can be predicted by interest rates, equity premia, dividends, etc. Merton suggests that the total demand for the risky asset is the demand of a single period mean variance maximizer, and demand as a hedge against unfavorable shifts in returns. The optimal portfolio rule has two components; myopic and non-myopic. In the Merton intertemporal paradigm 
myopic demand and hedging demand are additive. When the stochastic process is not IID, the portfolio holdings will change with time depending on the stochastic process chosen. With a mean reverting stochastic process, intertemporal hedging demands will not be zero. When returns are mean reverting, or when utility is not logarithmic, non-myopic portfolio choice includes hedging, or speculation on risk premium uncertainly, holding more or less of the risky asset at longer horizons. A dynamic non-myopic intertemporal portfolio strategy may hedge or speculate on expected return or mean-reversion risk, may be long or short risky assets, and may hold more of less risky assets at longer horizons. Kim and Omberg [13] show that a non-myopic portfolio strategy is optimal when the optimal portfolio in this revision period depends on the investment opportunity set in future revisions. The intertemporal optimal portfolio will be the same as the myopic portfolio only in the final revision period of the holding period.

The Merton [6] multi-period model maximizes investor's expected utility allowing continuous or periodic revision of optimal portfolios, to adapt to shifts in wealth, the investment opportunity set, and to the time left in the holding period. Non-myopic hedging demand is accomplished by holding negatively autocorrelated assets to hedge against intertemporal shifts in the efficient frontier. Intertemporal hedging (Merton [5]) lowers the variability of wealth. The size of intertemporal hedging demand for risky assets depends on the autocorrelations, or mean-reversions of returns and their lengths relative to the holding period.

\subsection{Hedging and Speculative Demand, Mean-Reversion Length, Holding Period, and Revision Frequency}

In the Merton model, mean-reversion is the only source of hedging demand in each revision period. Hedging demands arise in the long horizon problem with intermediate revision since investors can partially hedge against future changes in returns by deviating from their single period optimal portfolio. Serial covariance of returns induces hedging demands. When returns are predictable using a set of lagged state variables, multi-period optimizers' hedging demands will affect the composition of their equity portfolios. When there is predictability in the return process, a sequence of myopic solutions that ignore autocorrelation is not an optimal trading strategy. With predictability, the non-myopic investor will try to reduce risk by holding securities with mean-reversions that are out of phase, negatively correlated, or shorter than the holding period. Alternatively, the non-myopic investor may speculate by moving to lower risk assets in anticipation that the predictable mean-reversion will allow the high-risk asset to be purchased at a lower price in a subsequent revision. The non-myopic investor may also speculate based on expected return forecasts by holding high-risk assets with mean-reversion length longer than the holding period. Hedging and speculative demands depend on; forecasting variables, mean-reversion lengths (mean-reversion speeds), holding period, and revision frequency. Using a finite life investor, Lynch and Balduzzi [32] find that with predictable returns, reducing the revision frequency from monthly to quarterly cause both young and old 
investors to increase allocation to risky assets.

In a dynamic setting with mean reverting returns, the myopic demand for the risky asset decreases with an increase in risk aversion, while the intertemporal hedging demand for risky assets increases. As horizon shortens, the myopic demand generally increases and the hedging demand decreases. In the Merton model, hedging demand goes to zero in the last period of the investment horizon. Shorter-term mean-reversion or faster mean-reversion speed may result in lower hedging demand but may increase myopic demand. Hedging demand will be larger for longer-term mean-reversions, or slower mean-reversion speeds if the holding period is longer than the mean-reversion length.

Kim and Omberg [13] show that non-myopic strategic investment behavior includes unconditional risk premium hedging and speculation in the Merton model, when risk premiums follow mean reverting diffusion processes. Positive hedging demand occurs when investors with higher risk aversion hold more of the risky asset the longer the horizon. With positive hedging demand, the variance of terminal wealth is reduced by buying risky assets that deliver wealth when investment opportunities are poor. Positive hedging demands are the result of mean-reversion lengths equal to or shorter than the holding period. Hedging demands go to zero and conditional speculative demands for risky assets occur when the mean-reversion length is longer than the holding period. Positive or zero speculative demands for risky assets (with no short selling) are based on forecasts of risk premiums, or expected return. Mean-reversion increases demand for risky assets if the risk premium is positive. Optimal allocation to the risky asset will increases with investment horizon. If the risk premium is negative, hedging demand for risky assets will be zero or negative and there may be speculative demand for lower risk assets. Zero or negative hedging demand reduces total demand for risk assets. If investors expect future returns to become negative, they will have negative hedging demands and will short stocks to profit from the larger negative returns. Negative or zero speculative demand results when investors with low risk aversion speculate by holding less of the risky asset. Positive hedging demands, making the risky asset more attractive at longer horizons, are the result of unconditional negative autocorrelations in returns, or mean-reversions. The variance of returns grows less than linearly when returns are negatively autocorrelated. Kim and Omberg show that negative or zero hedging, or speculative demands (holding less risky assets the longer the horizon) result from unconditional positive autocorrelation or mean aversion in returns longer than the holding period.

\subsection{Problems with Intertemporal Solutions}

One problem with the Merton multi-period solution is the difficulty of modeling the conditional return distributions (mean, variance, and covariance) when they are time varying and predictable. State variables forecast conditional moments of return. Forecasting asset returns and state variables over long horizons is problematic. Intertemporal models frequently make the unrealistic assumption that 
investors know the predictive model and its parameters for the dynamics of asset returns and state variables. Predicting future returns or state variables at long horizons using term structures, or dividend yields is subject to large forecasting errors. When a statistical model such as the autoregressive is used, it imposes a structure on the dynamics of returns. Many studies allow the risk premium to follow Ornstein-Uhlenbeck process in order to capture stochastic mean-reversion effects (Kim and Omberg [13], Merton [5], Wachter [33]. In AR (1) models poor stock returns are correlated with better future returns so stocks can be used to hedge the variation in their future returns.

The optimal portfolio weights for Merton's dynamic portfolio choice problem are solutions to nonlinear partial differential equations (PDE). The solution is a trading strategy given by weights on each asset at each period and is frequently restricted to the choice between a risk-free and a risky asset. A state variable's stochastic process conditions returns and variances. The optimal weights are found by defining a dynamic programming problem assuming conditional expectation of moments in each period based on state variables (interest rates, or dividend yield, etc.) in the prior period. Backward recursion, assuming the investor's utility or value function, is used to solve the multi-period problem. Optimal dynamic policies for multi-period problems are found numerically, by stochastic dynamic programming, or by approximation using restrictive statistical models. Dynamic intertemporal models introduced additional estimation errors and instability to the Markowitz static approach. In the Merton model, long-term investors should revise their portfolios daily in response to state variables. Frequent portfolio adjustment adds a greater cost to this long-term model.

\section{Digital Portfolio Theory}

DPT is a theoretical and practical enhancement to the implementation of estimating efficient portfolios by using mean-reversion risks. Like MPT, DPT gives a single period solution. Like intertemporal portfolio choice, the DPT solution is non-myopic because it depends on holding period and estimates of mean-reversion risk levels. The discrete time signal processing of a discrete return process measures levels of mean-reversion risks. Unlike MPT, DPT does not assume any return distribution. DPT uses a nonparametric description of long-term risk. Unlike intertemporal portfolio choice, DPT does not forecast state variables or returns but uses forecasts of the long-term variance and its mean-reversion risk components. DPT uses low-frequency signal processing to describe mean-reversion risk levels of the unconditional variance. The discrete time Fourier transform gives a nonparametric description of the total variance and autocovariance by making a one-to-one transform of a finite length discrete return signal to a discrete risk spectrum. The digital return signal, $\tilde{r}[n]$ consists of a sequence of returns of length $T$,

$$
\tilde{r}_{j}[n], n=1,2,3, \cdots, T
$$


The square brackets indicate a digital process. The integer $n$ indicating the place in the sequence and $\tilde{r}_{j}[n]$ is the stochastic return at time $n \delta t$ for asset $j$. The observation horizon is $\delta t$. T gives the finite return signal length (bandwidth) but is not the holding period. DPT uses a finite duration signal, or finite bandwidth. Digital return signals are constructed from discontinuous prices and dividends. The finite discrete time, discrete frequency Fourier transform (DFT) of the low-frequency return signal gives the mean-reversion risk makeup of the original signal,

$$
R_{k j}=R_{j}[k \omega]=\sum_{n=1}^{T} \tilde{r}_{j}[n] \exp (-i[k \omega] n), k=1,2, \cdots, K=T / 2 \delta t
$$

$R_{k p}$ or $R_{j}[k \omega]$ represent the mean-reversion risk, or standard deviation of return of the kth mean reverting return component contained in the return signal. The $K$ mean-reversion risks describe the total variance of the original signal. The number of harmonic periods, $K=T / 2 \delta t$ depends on $\delta t$, the sampling interval, or observation horizon and the signal length, $T$. The angular frequency, $\omega=2 \pi / T$ depends on the signal length, $T . R_{k j}$ is the mean-reversion risk with mean-reversion length $p_{k}$

$$
p_{k}=T \delta t / k, k=1,2,, K=T / 2 \delta t
$$

The period, $p_{k}$ is the mean-reversion length of the kth mean-reversion. The lengths of the mean-reversions decrease harmonically. The $K$ mean-reversion variance components are uncorrelated, non-overlapping, and form a complete orthogonal set. The power spectrum or mean-reversion variance spectrum of the original signal consists of mean-reversion risks for $\mathrm{K}$ horizon lengths,

$$
\sigma_{j}^{2}=\operatorname{var}\left(\tilde{r}_{j}[n]\right)=\frac{1}{2} \sum_{k=1}^{K} R_{k j}^{2}
$$

The total single period variance of the return signal (1) is $1 / 2$ the sum of $K$ longer horizon variance components, $R_{k}^{2}$. Jones [3] [34] presents the derivation of DPT. The low-frequency discrete time, discrete frequency signal processing expression for the variance, covariance, and autocovariance, of a portfolio of $N$ assets is

$$
\sigma_{p}^{2}=\operatorname{var}\left(\tilde{r}_{p}[n]\right)=\frac{1}{2}\left(\sum_{k=1}^{K}\left(\sum_{j=1}^{N} w_{j} R_{k j} \cos \theta_{k m j}\right)^{2}+\left(\sum_{j=1}^{N} w_{j} R_{k j} \sin \theta_{k m j}\right)^{2}\right)
$$

where

$\sigma_{p}^{2}=\operatorname{var}\left(\tilde{r}_{p}[n]\right)$ the total single period variance of a portfolio of $N$ assets,

$\tilde{r}_{p}[n]=$ stochastic portfolio return in period $n, n=1,2, \cdots, T$,

$w_{j}=$ weight of asset $j$ in the portfolio,

$R_{k j}=$ the mean-reversion risk of period $p_{k}$-length returns of asset $j$,

$\theta_{k m j}=$ relative mean-reversion phase between asset $\mathrm{m}$ and asset $\mathrm{j}$, for the kth mean-reversion length,

$\cos \theta_{k m j}=$ correlation between asset $\mathrm{m}$ and asset $\mathrm{j}$, for the kth mean-reversion length, 
$R_{k j} \cos \theta_{k m j}=$ systematic or beta risk of the kth mean-reversion of asset $j$ relative to asset $m$,

$R_{k j} \sin \theta_{k m j}=$ unsystematic or alpha risk of the kth mean-reversion of asset $j$ relative to asset $m$,

$K=$ the number of mean-reversion lengths measured, $k=1,2, \ldots, K ; K=1 / 2 T$,

$T=$ the signal length (for example for $T=48$ months, $K=24$ ),

$p_{k}=$ kth mean-reversion length; $p_{k}=T \delta t / k, k=1,2, \cdots, K=T / 2 \delta t$,

$\delta t=$ sampling interval or revision length (1-month).

The single period total portfolio variance; $\sigma_{p}^{2}=\operatorname{var}\left(\tilde{r}_{p}[n]\right)$, is composed of $K$ mean-reversion variance contributions that describe the variance, covariance, and autocorrelation of the portfolio return process. These $K$ portfolio mean-reversion risks are uncorrelated and non-overlapping. DPT portfolio selection is more robust than time domain models because of the orthogonal nature of mean-reversion risks. For example, 1-year mean-reversion risk is independent of 3-month mean-reversion risk. In Equation (5) the portfolio variance uses the relative mean-reversion phases, $\theta_{k m i r}$ The correlation between assets is measure by the phase relative to the market index, or benchmark index. DPT uses index relative correlations rather than individual inter-asset correlations. The $K$ meanreversion risks are further composed of passive and active risk contributions. The cosine terms describe the systematic or beta risk and the sine terms the unsystematic or alpha risk. The cosine of the relative phase $\left(\cos \theta_{k m i j}\right)$ is the correlation between the security and a benchmark for the kth mean-reversion length. The objective of DPT is the same as MPT, to maximize expected portfolio return. Jones [35] shows how a relaxation of the portfolio variance (5) allows us to find efficient portfolios with an LP solution. We replace the MPT normal distribution variance constraint by multiple constraints on the contributions to one period variance; of long and short horizon returns, and passive (beta) and active (alpha) returns. The original Jones [3], long only, $D P T$ formulation is

Maximize

$$
E\left(\tilde{r}_{p}(t)\right)=\sum_{j=1}^{N} w_{j} E\left(\tilde{r}_{j}(t)\right)=\sum_{j=1}^{N} w_{j} \mu_{j}
$$

subject to: $4 \mathrm{~K}$ constraints $(K=24) \quad k=1,2,3, \cdots, K$

$$
\begin{gathered}
\sum_{j=1}^{N} w_{j} R_{k j} \cos \theta_{k m j} \leq c \beta_{k} \\
\sum_{j=1}^{N} w_{j} R_{k j} \cos \theta_{k m j} \geq-c \beta_{k} \\
\sum_{j=1}^{N} w_{j} R_{k j} \sin \theta_{k m j} \leq c \alpha_{k} \\
\sum_{j=1}^{N} w_{j} R_{k j} \sin \theta_{k m j} \geq-c \alpha_{k} \\
\sum_{j=1}^{N} w_{j}=1
\end{gathered}
$$




$$
w_{j} \geq 0, j=1,2,3, \cdots, N
$$

where $w_{j}$ is the weight of asset $j$ in the portfolio, $R_{k j}$ is the mean-reversion risk for the kth horizon length, or standard deviation of kth period returns of asset $j$. The $\mathrm{kth}$ phase-shift between index $\mathrm{m}$ and security j's returns is, $\theta_{k m ;}$. The k cross-correlations, $\theta_{k m p}$ define the multiple horizon correlation structure. $K$ is the number of horizon lengths measured $(k=1,2, \cdots, K)$ and is $1 / 2$ the signal length $\mathrm{T}$ (for example if $T=48$ months, $K=24$, ). $c \beta_{k}$ is the right hand side (RHS) constant that limits systematic or $\beta$-risk of mean-reversion length $p_{k} c \alpha_{k}$ is a RHS constant that limits unsystematic or $\alpha$-risk of the portfolio's mean-reversion risk with length $p_{k}$ DPT is a linear model and is stable for very large universes and long time periods. It allows control over the risk from long-term mean reverting components generating single period portfolio variance. Table 1 shows 24 mean-reversion risk components for a 48-month signal. For a 48-month signal length, DPT has $4 \times 24=96$ mean-reversion constraints. There are period lengths related to the calendar risk structure of the economy and there are period lengths that are non-calendar related. Table 2 shows the calendar related mean-reversions. A stationary calendar structure is the driving force or carrier for all risky asset return signals. Jones [36] finds calendar length components of risk are significantly different from a random walk for all individual US stocks' total and idiosyncratic variance.

The relaxed constraint set, (7) to (10), describes the long-term portfolio variance (5) that results in single period risk. These risk constraints are uncondi-

Table 1. Calendar and non-calendar mean-reversion lengths ${ }^{\mathrm{a}}$.

\begin{tabular}{|c|c|c|c|c|c|c|c|}
\hline $\mathrm{k}$ & $\begin{array}{c}\text { Period } \\
\mathrm{p}_{\mathrm{k}} \\
\text { (Months) }\end{array}$ & $\begin{array}{c}\text { Calendar } \\
\text { Based } \\
\text { Risk }\end{array}$ & $\begin{array}{c}\text { Calendar } \\
\text { Effect }\end{array}$ & $\mathrm{k}$ & $\begin{array}{c}\text { Period } \\
\mathrm{p}_{\mathrm{k}} \\
\text { (Months) }\end{array}$ & $\begin{array}{c}\text { Calendar } \\
\text { Based } \\
\text { Risk }\end{array}$ & $\begin{array}{c}\text { Calendar } \\
\text { Effect }\end{array}$ \\
\hline 1 & 48.0 & $4-\mathrm{yr}$ & Presidential & 13 & 3.7 & & \\
\hline 2 & 24.0 & $2-y r$ & Election & 14 & 3.4 & $8-y r$ & Business Cycle \\
\hline 3 & 16.0 & & & 15 & 3.2 & $16-y r$ & Long Term \\
\hline 4 & 12.0 & $1-\mathrm{yr}$ & Annual/Summer & 16 & 3.0 & $1 / 4-\mathrm{yr}$ & Quarter \\
\hline 5 & 9.6 & & & 17 & 2.8 & & \\
\hline 6 & 8.0 & $8-y r$ & Business Cycle & 18 & 2.7 & & \\
\hline 7 & 6.9 & & & 19 & 2.5 & & \\
\hline 8 & 6.0 & $1 / 2-y r$ & Sell in May & 20 & 2.4 & $1-\mathrm{mo}$ & Jan/Nov \\
\hline 9 & 5.3 & & & 21 & 2.3 & & \\
\hline 10 & 4.8 & $8-y r$ & Business Cycle & 22 & 2.2 & $8-y r$ & Business Cycle \\
\hline 11 & 4.4 & & & 23 & 2.1 & & \\
\hline 12 & 4.0 & $1-\mathrm{mo}$ & Jan/Nov & 24 & 2.0 & $1-\mathrm{mo}$ & Jan/Nov \\
\hline
\end{tabular}

aThere are calendar periods corresponding to long, intermediate, and short-term institutional event intervals with a 48-month signal length and monthly returns using low-frequency digital signal processing. The remaining periods are non-calendar. In the discrete Fourier transform (DFT) framework, the 24 mean-reversion lengths generate total 1-month risk. 
Table 2. Representing risk based on horizon lengths ${ }^{\mathrm{a}}$.

\begin{tabular}{cc}
\hline Period & Associated Calendar Effect \\
\hline 1 -month & January/November \\
1 -quarter & Quarter \\
6 -month & Sell in May \\
1-year & Annual/Summer \\
2-year & Election \\
4-year & Presidential \\
8-year & Business Cycle \\
16-year & Long Term \\
Noise & Random Walk \\
\hline
\end{tabular}

${ }^{a}$ Using digital signal processing (DSP) with a 4-year signal and a monthly observation horizon there are eight calendar mean-reversion lengths. These calendar mean-reversion lengths are associated with calendar effects. Multiple seasonal and cyclical effects as well as non-calendar effects such as white noise influence one-month variance.

tional; they do not change over time since mean-reversion lengths in all return process are assumed stationary. They describe the distribution of portfolio risk by mean-reversion lengths. As with MPT, the expected returns in the objective function of DPT may be estimated using historical means, or using multi-period forecasts based on state variables or past returns. With mean reverting returns the expected geometric mean can be used when the holding period is longer than one month. The DPT optimal portfolios depend on multiple horizon risks. DPT allows hedging, or speculative demands for multiple length mean-reversion risks. Holding mean-reversion risks with lengths equal to or shorter than the holding period will hedge unexpected changes in returns while reducing the variance of terminal wealth. We can hedge the risk of remaining unexposed to in-holding-period mean-reversions. For mean-reversion lengths longer than the holding period speculative demands will change the risk exposure of the optimal solution. Speculative demand may also apply to mean-reversion risks with lengths shorter than the holding period when the investor has a forecast of the pattern of the mean-reversion. The DPT formulation does not predict mean reverting turning points, or their cyclical phase, only the risk given the mean-reversion length. Cyclical or periodic speculation must be based on signals, or forecasts of expected returns or risk premiums that predict mean-reversion patterns.

DPT finds optimal single period policies that depend on intertemporal hedging and speculative demands for multiple length, unconditional, non-overlapping, mean-reversion risks. Covariance and autocovariance are estimated using low-frequency DSP. DPT solutions are non-myopic unless all return processes are white noise. In DPT, single period variance results from contributions to variance from longer horizon returns. Risk is constrained based on mean-reversion length, holding period, and desired exposure to active and passive risk. DPT does not utilize predictable expected return patterns because the phase is lost in 
taking the Fourier transform. The DPT paradigm assumes that the levels of risk that long-term mean reverting patterns generate are stable. DPT assumes no information about what patterns other than their length. By choosing appropriate values of mean-reversion risk RHS constants; $c \beta_{\mathrm{k}}$ and $c \alpha_{\mathrm{k}}$ in constraints (7) to (10), the linear DPT portfolio selection problem independently controls $\mathrm{K}$ horizon systematic and unsystematic mean-reversion risks. The kth risk constraint set (7) to (10) is statistically independent from the $\mathrm{k}+1$ constraint set. The risk contribution of 4-year returns to one-month risk is independent of the risk contribution of 2-year returns, or 1-year returns, etc. to one-month risk. The nonoverlapping variance contributions of multiple mean-reversion lengths are an essential feature of the frequency domain. There are four risk constraints for each mean-reversion length. The first two constraints (7) and (8) control the upper and lower bounds on systematic (beta) risk (cosine terms), and the second two risk constraints (9) and (10) control upper and lower bounds on unsystematic (alpha) risk (sine terms).

The relative phases, $\theta_{k m p}$ are estimated with a cross-spectral density between the security and the market index or benchmark returns. The phase-shifts, $\theta_{k m j}$ in constraint equations (7) to (10), give the relative lead or lag of the kth meanreversion length component between security $j$ and index $m$. The cosine and sine terms relate to the time domain. The cosine of the phase shift, $\theta_{k m j}$ is the correlation between $\mathrm{p}_{\mathrm{k}}$-length returns of the two processes,

$$
-1 \leq \rho_{k m j}=\cos \theta_{k m j} \leq 1
$$

where $\rho_{k m j}$ is correlation of security $j$ with the index portfolio m's kth periodic returns. For example with $k=4, \rho_{4 m j}$ equals the correlation between all annual returns of security $j$ and index $m$. The $\cos \theta_{k m j}$ is the in-phase or systematic component and $\sin \theta_{k m j}$ is called the quadrature or unsystematic component where

$$
\cos ^{2} \theta_{k m j}+\sin ^{2} \theta_{k m j}=1, k=1,2, \cdots, K
$$

The phase-shift relative to another signal, particularly a strong signal such as the benchmark index is estimated at higher significance than inter-asset correlations used in MPT.

The normative $D P T$ model prescribes what investors should do in the presence of mean-reversion risk in each revision period of their holding period. There are multiple single period efficient frontiers, one for each mean-reversion length. The statistical independence of the $\mathrm{K}$ mean-reversion risk components allows for multiple fund separation. Because the $\mathrm{K}$ mean-reversion risks are orthogonal, uncorrelated, and non-overlapping, K-fund separation applies. DPT implies positive models; calendar based or horizon based CAPM, and an autocovariance APT (Jones [34]) with expected return and risk premiums changing in time to provide compensation for the multiple mean-reversion horizon betas.

\subsection{Digital Portfolio Theory is Non-Myopic}

The DPT mean-reversion risk model is non-myopic. The one period solution includes the variance contributed by long-term mean-reversions. The DPT solu- 
tion allows the application of hedging and speculative demands based on investor's holding period. Investors with shorter holding periods will have zero hedging demand for the risk of longer length mean-reversions. On the other hand, investor's with longer holding periods should have larger hedging demands since more mean-reversion lengths will be within the holding period. Reducing risk by holding a larger portion of a portfolio in high risk-return assets the longer the investment horizon is called time diversification. In addition to zero hedging demand for mean-reversion risks longer than the holding period, investors will have zero hedging demand for non-calendar length mean-reversion risks. Levels of mean-reversion active and passive risk are controlled for the 24 mean-reversion lengths given in Table 1. By tightening or losing the RHS constants $c \beta_{k}$ in Equations (7) and (8), the level of systematic risk of mean-reversion length $p_{k}$ is constrained in the optimal portfolio. The RHS constants $c \alpha_{k}$ in equations (9) and (10) limit levels of unsystematic risk.

Take the example of a particular investor with a holding period of four years. The investor needs a portfolio to hold over the first month. For each the 24 mean-reversion lengths in Table 1, the investor must decide to hedge, speculate, or stay myopic for passive and active risk. The myopic solution to DPT will result when all RHSs are set equal $\left(c \beta_{k}=c \alpha_{k}\right)$ for all $\mathrm{k}$. This will constrain all risk contributions to the portfolio variance equally. When all return processes in the economy are random walks this myopic solution will be optimal. The random walk process has no mean-reversion. The random walk variance has equal risk contributions at all mean-reversion lengths. DPT finds a myopic efficient frontier by changing the constant RHS value for all constraints. The myopic investor will select the efficient portfolio that satisfies his or her risk preference. Non-calendar mean-reversion lengths have not been found to be different from a random walk (Jones [36]). Therefore the myopic solution will be appropriate for the non-calendar constraints. The investor can hedge by taking more risk for calendar mean-reversion lengths within the holding period. The four year investor will have positive hedging demand for 4-year, 2-year, 1-year, 1/2-year, 1/4-year, and 1-month mean-reversion risk. The investor will have zero hedging demand for non-calendar risk and zero hedging demand for 8-year and 16-year risk since they are longer than the four year holding period. To hedge we can increase the level of 4-year, 2-year, 1-year, 1/2-year, 1/4-year, and 1-month mean-reversion risk by increasing these constraint RHSs. RHSs can be increased for $c \beta_{k}$ and $c \alpha_{k}$ for $\mathrm{k}=1,2,4,8,16$ and for $\mathrm{k}=12,20$, and 24 . Since we are sampling monthly, 1month risk is reflected in harmonics of 2-months, 2.4-months and 4-months. Hedging will reduce our risk in the holding period while increasing expected return. If the investor prefers a passive portfolio, $c \beta_{k}$ can be set higher than $c \alpha_{k}$ Since DPT assumes no short selling, the DPT optimizer cannot satisfy negative hedging demand.

Speculative demand results when the investor has a forecast of the phase of mean reverting returns for a particular mean-reversion length. Conditional speculative demand for the risk of a particular mean-reversion length will depend 
on forecasts of patterns within a given mean-reversion length. The investor can use the mean-reversion constraints (7) to (10) to speculate. For example, if the investor believes an 8-year market cycle is currently at the bottom, he or she can increase 8-year mean-reversion risk by increasing $c \beta_{k}$ and $c \alpha_{k}$ for $\mathrm{k}=6,10,14$ and 22. Since the signal length we are using is four years, 8 -year risk is reflected in the shorter harmonics; 2.2-month, 3.4-month, 4.8-month, and 8-month. Similarly if we believe 6-month mean-reversion is currently at a top we can speculate by tightening $c \beta_{k}$ and $c \alpha_{k}$ for $\mathrm{k}=8$. This will force 6-month risk out of the portfolio so that we can buy 6-month risk after returns fall.

At the end of the month the investor must find a new solution for the next month. The holding period has shortened to three years and 11 months. We can no longer use 4-year risk to hedge. We continue to hedge using 2-year, 1-year, 1/2-year, 1/4-year, and 1-month risk. As the holding period shortens fewer mean-reversion lengths can be used to hedge. In the last month of the holding period only 1-month risk is available to hedge. The question of how much mean-reversion risk we should use to hedge remains an open question.

\subsection{DPT Solutions}

The DPT model adopts the single index correlation structure proposed by Markowitz [37] but in 24 independent time horizon dimensions rather than in onedimension. Measuring the covariance structure relative to a benchmark process is more stable and significant than the MPT covariance matrix. DPT solutions do not require matrix inversion so there is less magnification of estimation error. With no squared, or cross product decision variables and no matrix inversion, estimation error is not magnify using DPT. DPT solutions are stable and consistent with equilibrium pricing models. The solutions are stable because the longterm correlations and autocorrelations are estimated relative a strong signal such as a market index rather than relative to other individual assets. DPT solutions are consistent with asset pricing models because cross correlations are estimated relative to a market index and the solutions are linear. DPT has a linear not a quadratic formulation and solves quickly for large universes. The DPT efficient portfolio weights are more reliable and stable than for MPT. In addition, the total number of terms used in DPT grows linearly with universe size while the terms grow exponentially in MPT. The static LP DPT solution will not amplify estimation error even for large universe sizes. The DPT model includes more information about return processes by including long horizon mean-reversion variances. DPT accommodates active and passive strategies, and non-myopic hedging and speculative strategies to benefit from multiple mean-reversion risks in each investor's holding period.

Because DPT is a linear programming problem, integer variables can be added to include options positions, fixed trading costs, and portfolio size constraints (e.g. Jones [34] [38] [39]). DPT like MPT treats all parameters as known. The size of portfolio solutions in MPT and DPT are relatively small because the models assume parameters are known without estimation error. We can opti- 
mally increase portfolio size to diversify estimation error in DPT. Optimal larger portfolio size portfolios can be found using integer variables. Adding integer variables to control size in MPT results in a problem that is NP hard while adding integer variables to control size in DPT is straightforward.

The signal processing representation of portfolio variance avoids the problem of over-lapping mean-reversions encountered in time domain models. The level of confidence is higher because low-frequency signal processing provides robust non-overlapping estimates of periodic mean-reversion risks. DPT allows geometric mean, growth optimal, or momentum objective functions. It allows additional constraints such as; options constraints, shortfall constraints, solvency constraints, and fundamental constraints as well as other portfolio management constraints (e.g. Jones [34] Broadie [40]).

DPT assumes an observation horizon and revision period of one month. Shorter estimation horizons are not possible using low-frequency digital signal processing solutions. While the Merton model suggests portfolio revision daily, a revision frequency shorter than one month is not meaningful using DPT. Longer observation periods such as quarterly or yearly do not provide enough time series data to estimate DPT parameters reliably. Practical implementation DPT requires longer return histories than are often considered relevant for myopic MPT, or high-frequency trading. Long time series are required to measure long horizon autocorrelations. While it is common practice to estimate parameters of MPT using five years of data, DPT requires a minimum of 16 years of monthly returns. We can estimate long-term mean-reversion variance contributions to single period risk at higher significance with longer time series. In addition, longer history allows measurement of longer period mean-reversion risks.

MPT is a short-term volatility model while DPT is a long-term variance model. In MPT returns are independently distributed over time (IID), or constant and stationary resulting in myopic solutions. The random walk model suggests that shorter-term volatility (hourly or daily) generates one-month risk while the DSP model suggests that variances of multiple longer-term holding period returns generate one-month risk. While MPT researchers attempt to add tail risk to account for mean-reversion, DPT explicitly, accounts for mean-reversions up to sixteen years and can be formulated to include longer mean-reversions. In the DPT paradigm, stock returns are stationary but can have multiple length mean reverting patterns. Glover and Jones [41] compare DPT and MPT solutions and develop an algorithm to find MPT solutions using DPT.

Most intertemporal portfolio choice studies do not allow the multi-period investor to differentiate using a large universe of assets. Multiple period models are not able to identify buy-and-hold allocations to specific styles, sectors, or securities. Non-myopic single period DPT is able to identify buy-and-hold allocations to specific assets classes, or securities, over alternative horizons within optimal portfolios. The DPT model does not require conditioning predictive state variables or forecasts of expected returns but does allow intertemporal hedging by incorporating forecasts of unconditional longer-term mean-reversion variances. 
While MPT and intertemporal theory evolved into volatility models. In lowfrequency DPT, the shortest estimation period possible is a monthly period. DPT is a long horizon stationary variance model. By quantifying the risk of unconditional mean-reversions of different lengths, DPT can satisfies hedging and speculative demands that are consistent with the investor's holding period and expectations.

\section{Conclusions}

MPT assumes investors make decisions myopically in a static single period framework assuming no mean-reversion. Intertemporal portfolio choice assumes investors make intertemporal decisions dynamically based on conditional mean-reversions. DPT assumes investors make long-horizon decisions dynamically using static single period solutions that incorporate unconditional meanreversion risks. The DPT solution satisfies both myopic demand generated by the return maximizing objective and the covariance of returns, and satisfies nonmyopic demand generated by hedging and speculative demand for mean-reversion risks of different lengths in relation to the holding period.

DPT is linear with a linear programming solution, MPT requires quadratic programming, and intertemporal portfolio choice requires dynamic programming, or numerical methods. MPT does not provide for predictable time variation in the investment returns and the quadratic solution is ill-conditioned and unstable. Continuous-time portfolio choice provides useful understanding of strategic asset allocation in terms of hedging and speculative demands in the presence of mean-reversion. It is useful for daily revisions over yearly or longer holding periods, or monthly revisions for holding periods longer than twenty years. Intertemporal portfolio choice is hard to solve, is restricted to maximum utility formulations, and is limited to small asset universes. Intertemporal portfolio choice is difficult to implement because long-term forecasts of the dynamics of state variables and their relation to asset returns is subject to considerable uncertainty. The DPT solution includes hedging and speculative demand for multiple mean-reversion length risks and has a stable solution based on stationary mean-reversion variance. The DPT long-horizon model does not require forecasts of state variables or risk premiums but uses long-term forecasts of unconditional autocorrelations to allow multiple period strategic decisions in the single period solution. DPT uses long-term mean-reversion return variances to describe monthly risk. Investors will have zero hedging demand for mean-reversion risks with lengths longer than their holding period and zero hedging demand for non-calendar length mean-reversion risks. The application of lowfrequency digital processing in DPT gives investors a framework and effective model for constructing long-term portfolios in single period solutions. A better understand of DPT will help portfolio theory move from the age of volatility to the digital age of long-term reliability and stability.

MPT and intertemporal portfolio choice have serious theoretical and practical weakness. DPT improves on some of these shortcomings by incorporating 
long-term risk in a linear optimization. The main innovation and contribution of this research is to provide a single period solution that gives investors control over their exposure to the long-term distribution of portfolio returns as well as to passive and active portfolio returns. It provides a long horizon risk model and its linear formulation offers a significant potential for further innovation. DPT adds financial theory as a new field of digital technology. DPT is limited to long-term stationary mean-reversion components of risk. It does not capture short-term black swan risk. The method depends on using monthly time signals. Further research is needed to clarify how levels of hedging demand and speculative demand for mean-reversion risks affect long-term optimal solutions over various holding periods. DPT can be empirically tested to shed light on these and other issues.

\section{Acknowledgements}

I am grateful for the comments of Peter L. Bernstein, Dimitris Bertsimas, Mark N. Broadie, John Y. Campbell, John S. Cone, George B. Dantzig, Fred W. Glover, Clive W.J. Granger, Rustam Ibragimov, Steven M. Kay, Andrew W. Lo, David G. Luenberger, Harry M. Markowitz, Richard O. Michaud, Merton H. Miller, William T. Moore, Panos M. Pardalos, Stephen A. Ross, James Stock, Mark Rubinstein, Paul A. Samuelson, Charles S. Tapiero, John N. Tsitsiklis and Israel Zang. All errors are the responsibility of the author.

\section{References}

[1] Markowitz, H.M. (1952) Portfolio Selection. Journal of Finance, 7, 77-91. https://doi.org/10.1111/j.1540-6261.1952.tb01525.x

[2] Merton, R.C. (1969) Lifetime Portfolio Selection under Uncertainty: The Continuous-Time Case. Review of Economics and Statistics, 51, 247-257. https://doi.org/10.2307/1926560

[3] Jones, C.K. (2001) Digital Portfolio Theory. Computational Economics, 18, 287 316. https://doi.org/10.1023/A:1014824005585

[4] Samuelson, P.A. (1969) Lifetime Portfolio Selection by Dynamic Stochastic Programming. Review of Economics and Statistics, 51, 239-246. https://doi.org/10.2307/1926559

[5] Merton, R.C. (1971) Optimum Consumption and Portfolio Rules in a ContinuousTime Model. Journal of Economic Theory, 3, 373-413.

[6] Merton, R.C. (1973) An Intertemporal Capital Asset Pricing Model. Econometrica, 41, 867-887.

[7] Jones, C.K. (2008) Calendar Based Mean Reversion Risk and Digital Signal Processing. SSRN Working Paper. http://papers.ssrn.com/sol3/papers.cfm?abstract $\mathrm{id}=1361886$

[8] Campbell, J.Y. and Viceira, L.M. (1999) Consumption and Portfolio Decisions When Expected Returns Are Time Varying. Quarterly Journal of Economics, 114, 433-495. https://doi.org/10.1162/003355399556043

[9] Brandt, M.W. (1999) Estimating Portfolio and Consumption Choice: A Conditional Euler Equation Approach. Journal of Finance, 54, 1609-1645.

https://doi.org/10.1111/0022-1082.00162 
[10] Ait-Sahalia, Y. and Brandt, M.W. (2001) Variable Selection for Portfolio Choice. Journal of Finance, 56, 1297-1351. https://doi.org/10.3386/w8127

[11] Barberis, N. (2000) Investing for the Long Run When Returns Are Predicable. Journal of Finance, 55, 225-264. https://doi.org/10.1111/0022-1082.00205

[12] Brennan, M.J., Schwartz, E.S. and Lagnado, R. (1997) Strategic Asset Allocation. Journal of Economics Dynamics and Control, 21, 1377-1403.

[13] Kim, T.S. and Omberg, E. (1996) Dynamic Nonmyopic Portfolio Behavior. Review of Financial Studies, 9, 141-161. https://doi.org/10.1093/rfs/9.1.141

[14] Liu, J. (2007) Portfolio Selection in Stochastic Environments. Review of Financial Studies, 20, 1-39. https://doi.org/10.1093/rfs/hhl001

[15] Campbell, J.Y. and Viceira, L.M. (2002) Strategic Asset Allocation: Portfolio Choice for Long-Term Investors. Oxford University Press, Oxford. https://doi.org/10.1093/0198296940.001.0001

[16] Balduzzi, P. and Lynch, A.W. (1999) Transaction Costs and Predictability: Some Utility Cost Calculations. Journal of Financial Economics, 52, 47-78.

[17] Chacko, G. and Viceira, L.M. (1999) Dynamic Consumption and Portfolio Choice with Stochastic Volatility in Incomplete Markets. NBER Working Paper No. 7377. https://doi.org/10.3386/w7377

[18] Lynch, A.W. (2001) Portfolio Choice and Equity Characteristics: Characterizing the Hedging Demands Induced by Return Predictability. Journal of Financial Economics, 62, 67-130.

[19] Brandt, M.W. and Santa-Clara, P. (2006) Dynamic Portfolio Selection by Augmenting the Asset Space. Journal of Finance, 61, 2187-2217.

https://doi.org/10.1111/j.1540-6261.2006.01055.x

[20] Merton, R.C. and Samuelson, P.A. (1974) Fallacy of the Log-Normal Approximation to Optimal Portfolio Decision-making over Many Periods. Journal of Financial Economics, 1, 67-95.

[21] Perold, A.F. (1984) Large-Scale Portfolio Optimization. Management Science, 30, 1143-1160. https://doi.org/10.1287/mnsc.30.10.1143

[22] Michaud, R.O. (1989) The Markowitz Optimization Enigma: Is “Optimized” Optimal? Financial Analysts Journal, 45, 31-42. https://doi.org/10.2469/faj.v45.n1.31

[23] Green, R.C. and Hollifield, B. (1992) When Will Mean-Variance Efficient Portfolios Be Well Diversified? Journal of Finance, 47, 1785-1809. https://doi.org/10.1111/j.1540-6261.1992.tb04683.x

[24] Frost, P.A. and Savarino, J.E. (1988) For Better Performance: Constrain Portfolio Weights. Journal of Portfolio Management, 15, 29-34.

https://doi.org/10.3905/jpm.1988.409181

[25] Jagannathan, R. and Ma, T. (2003) Risk Reduction in Large Portfolios: Why Imposing the Wrong Constraints Helps. Journal of Finance, 58, 1651-1684. https://doi.org/10.1111/1540-6261.00580

[26] Jobson, J.D. and Korkie, B. (1980) Estimation of Markowitz Efficient Portfolios. Journal of the American Statistical Association, 75, 544-554. https://doi.org/10.1080/01621459.1980.10477507

[27] Black, F. and Litterman, R. (1992) Global Portfolio Optimization. Financial Analysis Journal, 48, 28-43. https://doi.org/10.2469/faj.v48.n5.28

[28] Jobson, J.D. and Korkie, B. (1981) Putting Markowitz Theory to Work. Journal of Portfolio Management, 7, 70-74. https://doi.org/10.3905/jpm.1981.408816

[29] Ledoit, O. and Wolf, M. (2004) Honey, I Shrunk the Sample Covariance Matrix. 
Journal of Portfolio Management, 30, 110-119.

https://doi.org/10.3905/jpm.2004.110

[30] Treynor, J.L. and Black, F. (1973) How to Use Security Analysis to Improve Portfolio Selection. Journal of Business, 46, 66-86. https://doi.org/10.1086/295508

[31] Pastor, L. and Stambaugh, R.F. (2000) Comparing Asset Pricing Models: An Investment Perspective. Journal of Financial Economics, 56, 335-381.

[32] Lynch A.W. and Balduzzi, P. (2000) Predictability and Transaction Costs: The Impact on Rebalancing Rules and Behavior. Journal of Finance, 55, 2285-2310. https://doi.org/10.1111/0022-1082.00287

[33] Wachter, J.A. (2002) Portfolio and Consumption Decisions under Mean-Reverting Returns: An Explicit Solution for Complete Market. Journal of Financial and Quantitative Analysis, 37, 63-91. https://doi.org/10.2307/3594995

[34] Jones, C.K. (1992) Portfolio Management: New Model for Successful Investment. McGraw-Hill, London.

[35] Jones, C.K. (1983) Portfolio Selection in the Frequency Domain. American Institute of Decision Sciences Proceedings November, 67-70.

[36] Jones, C.K. (2004) Calendar Based Risk, Firm Size, and the Random Walk Hypothesis. SSRN Working Paper. http://ssrn.com/abstract=639683

[37] Markowitz, H. M. (1959) Portfolio Selection: Efficient Diversification of Investments. John Wiley \& Sons, New York.

[38] Jones, C.K. (2007) Fixed Trading Costs, Signal Processing and Stochastic Portfolio Networks. European Journal of Industrial Engineering, 1, 5-21. https://doi.org/10.1504/EJIE.2007.012651

[39] Jones, C.K. (2013) Portfolio Size in Stochastic Portfolio Networks Using Digital Portfolio Theory. Journal of Mathematical Finance, 3, 280-290. https://doi.org/10.4236/jmf.2013.32028

[40] Broadie, M. (1994) Portfolio Management: New Models for Successful Investment Decisions. Journal of Finance, 49, 361-364. https://doi.org/10.2307/2329151

[41] Glover, F. and Jones, C.K. (1988) A Stochastic Generalized Network Model and Large-Scale Mean-Variance Algorithm for Portfolio Selection. Journal of Information and Optimization Sciences, 9, 299-316.

https://doi.org/10.1080/02522667.1988.10698932

\section{Submit or recommend next manuscript to SCIRP and we will provide best} service for you:

Accepting pre-submission inquiries through Email, Facebook, LinkedIn, Twitter, etc. A wide selection of journals (inclusive of 9 subjects, more than 200 journals)

Providing 24-hour high-quality service

User-friendly online submission system

Fair and swift peer-review system

Efficient typesetting and proofreading procedure

Display of the result of downloads and visits, as well as the number of cited articles

Maximum dissemination of your research work

Submit your manuscript at: http://papersubmission.scirp.org/

Or contact ajibm@scirp.org 